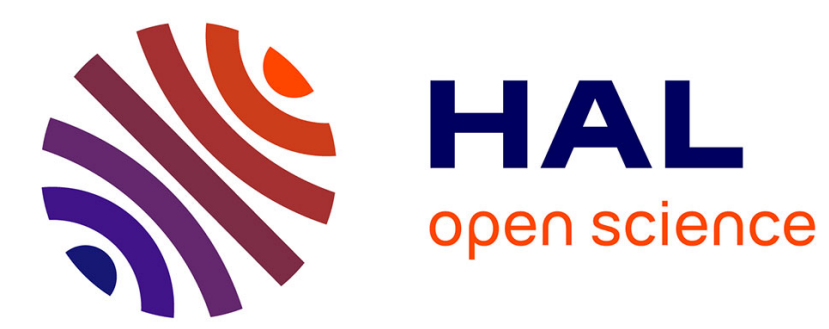

\title{
An On-Line Monitoring System of Crop Growth in Greenhouse
}

\author{
Wu Lixuan, Sun Hong, Li Minzan, Zhang Meng, Zhao Yi
}

\section{To cite this version:}

Wu Lixuan, Sun Hong, Li Minzan, Zhang Meng, Zhao Yi. An On-Line Monitoring System of Crop Growth in Greenhouse. 8th International Conference on Computer and Computing Technologies in Agriculture (CCTA), Sep 2014, Beijing, China. pp.627-637, 10.1007/978-3-319-19620-6_70 . hal01420281

\section{HAL Id: hal-01420281 \\ https://hal.inria.fr/hal-01420281}

Submitted on 20 Dec 2016

HAL is a multi-disciplinary open access archive for the deposit and dissemination of scientific research documents, whether they are published or not. The documents may come from teaching and research institutions in France or abroad, or from public or private research centers.
L'archive ouverte pluridisciplinaire HAL, est destinée au dépôt et à la diffusion de documents scientifiques de niveau recherche, publiés ou non, émanant des établissements d'enseignement et de recherche français ou étrangers, des laboratoires publics ou privés. 


\title{
An on-line monitoring system of crop growth in greenhouse
}

\author{
Wu Lixuan ${ }^{1, \mathrm{a}}$, Sun Hong ${ }^{1, \mathrm{~b}}$, Li Minzan ${ }^{1, \mathrm{c}}$, Zhang Meng ${ }^{2, \mathrm{~d}}$, Zhao $\mathrm{Yi}^{2, \mathrm{e}}$ \\ ${ }^{1}$ Key Laboratory of Modern Precision Agriculture System Integration Research, China \\ Agricultural University, Beijing 100083, China; \\ ${ }^{2}$ Key Laboratory of Agricultural information acquisition technology, Ministry of Agriculture, \\ Beijing 100083, China; \\ a zhwlx22@163.com, ${ }^{b}$ sunhong@cau.edu.cn, ${ }^{\mathrm{c}}$ pact@cau.edu.cn, ${ }^{\mathrm{d}}$ caupac_zm@cau.edu.cn, \\ ${ }^{\mathrm{e}}$ YiZhao@cau.edu.cn
}

\begin{abstract}
It is significant to estimate the growth of crops during their growth stages rapidly and non-destructively, the spectral analysis technology could satisfy the requirement and it is one of the important techniques to support in the precision agriculture management. In the article, a system was developed for estimating the growth of crops in greenhouse, which was based on spectrum and WSN (wireless sensor network) technology. The system consisted of three parts: the intelligent sensor nodes, intelligent gateway and the software in remote server. The ZigBee wireless communication modules were embedded in the intelligent sensor nodes and gateway to create WSN. The intelligent sensor nodes contained four optical channels, which allowed these instruments work at the wavelengths of 550,650, 766 and $850 \mathrm{~nm}$. They were used to collect spectral data and transmit to the intelligent gateway by ZigBee wireless network. The intelligent gateway worked as a coordinator of the whole wireless network waiting for sensor nodes to join in and receiving all the spectral data from sensor nodes, and transmitting the data to remote server by GPRS. The software in remote server analyzed the spectral data, calculated the NDVI (Normalized Difference Vegetation Index), by which, the growth of the plants can be detected effectively. In order to test the effectiveness and stability of the system, the strawberry of greenhouse was detected. Several experiments were carried out in the greenhouse of ZhuoZhou Experiment Station in Hebei. Results showed that the canopy spectral data of strawberries could be acquired by the intelligent sensor nodes stably, and the intelligent gateway could connect the sensor nodes and the remote server effectively. The spectral data can be received by the remote server normally as well. The system provides a kind of technical support and theoretical basis for crop growth estimation in greenhouse.
\end{abstract}

Keywords: spectral analysis, WSN, crop growth, greenhouse, ZigBee

\section{Introduction}

Evaluating crop growth status is one of important steps for the plant monitoring and predicting yield [1]. Nowadays, the chlorophyll and nitrogen content are generally selected as nutrient indicators to monitor crop growth status. At present, the most 
common way to get the chlorophyll and nitrogen content is by chemical analysis method in a laboratory, which is expensive, complicated and time-consuming [2-3]. Previous research revealed that nitrogen had a great effect on the chlorophyll content of the crop leaves, and could further cause a change of the spectral reflectance of the crop canopy [4]. And it was the theoretical basis for obtaining biochemical parameters of crops by spectral methods, which made it possible to use spectroscopy to estimate nitrogen content.

In recent years, researches were carried out on variation of crop canopy reflectance spectra and nitrogen sensitive band selection [5]. Simultaneously, a variety of portable crop canopy detectors based on vegetation index had been developed. The Green Seeker, developed by Marvin et al [6], collecting the crop canopy reflectance in the red and near infrared, by which, they can calculate the NDVI (Normalized Difference Vegetation Index) to analyze crop growth. Ni et al [7] (2013) developed a portable crop growth estimator by detecting the crop canopy reflectance at the wavelength of $710 \mathrm{~nm}$ and $820 \mathrm{~nm}$, then calculating vegetation index. Zhang et al [8] (2006) developed a handheld spectral instrument to estimate the growth status of the crop in a greenhouse using optical fibers. Li et al [9-10] (2009, 2012) developed two generations of crop growth detector, working at red and near infrared band, and based on this, they developed a 4-waveband crop growth detection sensor. Sui et al [11] (2005) developed a device for detecting nitrogen status in cotton plants by measuring the spectral reflectance of the cotton canopy at four wavebands (blue, green, red, and NIR). However, these instruments mentioned above cannot achieve the real-time detection of crop growth.

Therefore, in this article, a system for crop growth estimation in greenhouse was developed, which consisted of the intelligent sensor nodes, intelligent gateway and the software in remote server. The system can detect the crop canopy spectral data in greenhouse at 4-waveband, and then calculate the NDVI, by which, the growth of the plants can be detected effectively. The system provides a kind of technical support and theoretical basis for crop growth estimation in real time.

\section{Development for crop growth estimation system}

\subsection{Structure of crop growth estimation system}

The system was made up of intelligent sensor nodes, intelligent gateway and software in remote server. It was shown in Fig.1. Several intelligent sensor nodes connected with the intelligent gateway based on ZigBee wireless sensor network (WSN). On one hand, as the coordinator of the WSN, the intelligent gateway set up WSN, waiting for sensor nodes to join in, and receiving spectral data detected by the sensor nodes, on the other hand, the gateway connected with the remote server, transmitting spectral data to the remote server by GPRS. Meanwhile, the software in the remote server analyzed and processed the spectral data, and then calculated NDVI. The intelligent sensor nodes consisted of an optical part and a circuit part. The optical part contained four optical channels at the wavelengths of 550,650, 766 and $850 \mathrm{~nm}$ respectively. 
Since the system used sunlight as light source, the sunlight intensity should be measured as well besides measuring the crop canopy reflectance spectra.

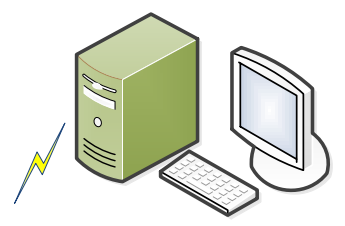

The software in remote server

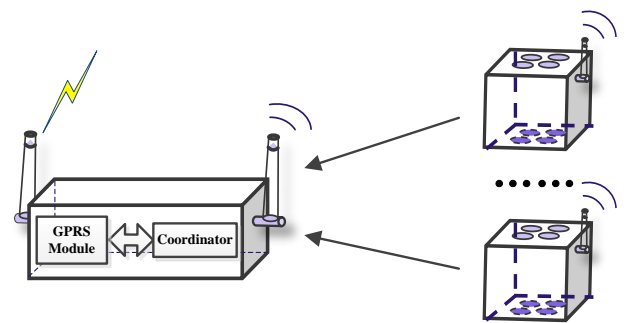

The intelligent gateway The intelligent sensor nodes

Fig. 1. Structure of the system of crop growth estimation

\subsection{Development of the intelligent sensor nodes}

The intelligent sensor nodes were designed to collect optical signal, realize photoelectric conversion, process and amplify electrical signal, send data etc. As an example shown in Fig.1, Fig.2 illustrated the block diagram of an intelligent sensor node. The whole sensor node mainly included an optical system, hardware circuits and software design.

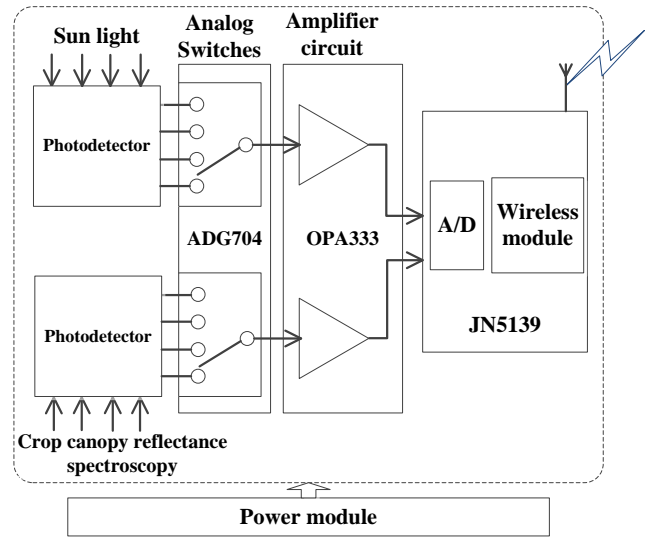

Fig. 2. Block diagram of intelligent sensor node 


\subsubsection{Development of optical system}

The whole optical system was designed with two parts: four optical channels to collect sunlight signal, and the other four optical channels to collect the crop canopy reflectance spectra signal. Each channel had the same structure, which mainly consisted of the convex lens, filters, photoelectric detector and mechanical enclosure. The convex lens had a $12.5 \mathrm{~mm}$-diameter and $12.5 \mathrm{~mm}$-focal-length. The center wavelengths of the filters were: $550 \mathrm{~nm}, 650 \mathrm{~nm}, 766 \mathrm{~nm}$ and $850 \mathrm{~nm}$, the bandwidth was $20 \mathrm{~nm}$. And all the photodiodes were PIN-Si photodiode which has many advantages, including a wide response range, high sensitivity and fast response.

\subsubsection{The structure of hardware circuits}

The hardware circuits of one single intelligent sensor node were mainly made up of three parts: the control unit, the signal processing unit and the power management unit. As the MCU of the control unit, a JN5139 wireless module (Jennic Co. UK), embedded ZigBee wireless communication protocol, has many advantages, including low power consumption, inexpensive and fully compatible with IEEE802.15.4. This microcontroller included a 4-input 12-bit A/D converter unit, meeting the requirements of the signal acquisition. And JN5139 Integrated $16 \mathrm{MHz} 32 \mathrm{bit}$ RISC MCU core, high-performance 2.4GHz IEEE 802.15.4 transceiver, 192KB ROM and $96 \mathrm{kB}$ RAM. Thus, the MCU provided a versatile low cost solution for wireless sensor network applications.

The signal processing unit mainly processed the photoelectric signal detected by the photoelectric detector. Firstly, a 4:1 time sharing analog multiplex chip ADG704 was applied to select four optical signals. Secondly, an OPA333 amplifier, which had the properties of high-precision, low quiescent current and low power consumption, was chosen to amplify the optical signal selected by ADG704. The output of the OPA333 was sent to the MCU, where the analog signal can be converted to digital signal in A/D converter.

The power management unit ensured the entire sensor node work normally. In this development, a $3.7 \mathrm{~V}, 1800 \mathrm{mAh}$ rechargeable lithium battery was chosen as an external power supply. And under the effect of the voltage converter chip SP6201, which had the properties of high-precision output voltage, low-power-consumption. The input voltage was converted into $3.3 \mathrm{~V}$ for other functional modules. Meanwhile, a $4.2 \mathrm{~V}$ power adapter was used to recharge the lithium battery. So the intelligent sensor node can work normally and steadily for a long time, meeting the requirements using in greenhouse.

\subsubsection{Software in intelligent sensor nodes}

Each sensor node was the end device in this ZigBee WSN and shared the same workflow. The flow chart of the software in the sensor node was illustrated in Fig.3. Once started, the sensor was initialized and the data were collected automatically with a certain sampling frequency. By setting the address of analog switch, the sensor selected the appropriate channel and collected data. Data acquisition of each channel 
was repeated for 10 times and averaged. When data collections of all the channels were completed, the data were sent to the coordinator via wireless module embedded in JN5139. Once finished, another five minutes was started for the next round of measurement and acquisition.

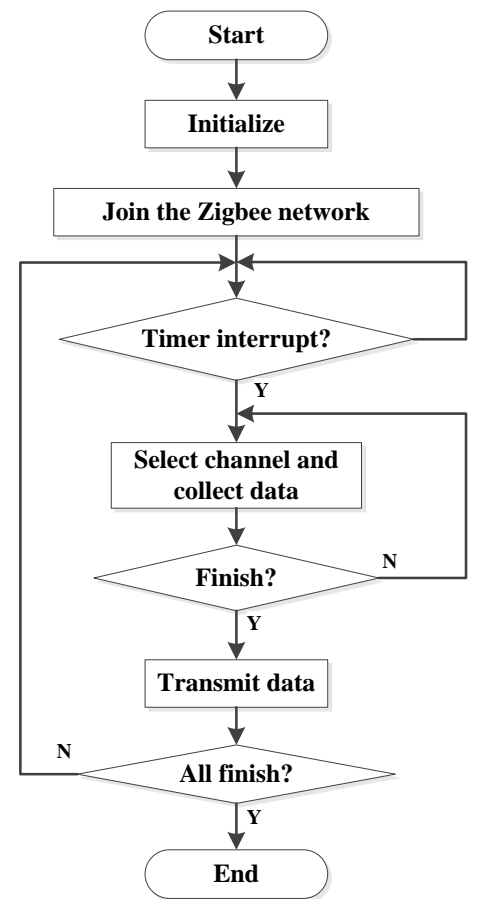

Fig. 3. Flow chart of software in sensor node

\subsection{Development of intelligent gateway}

The intelligent gateway was mainly consisted of two parts: the coordinator and the GPRS module. The coordinator was designed to set up wireless sensor network, waiting for intelligent sensor nodes to join in, receiving optical data, and then transmitting data to the remote server via the GPRS module. The structure of the intelligent gateway is illustrated in Fig.4.

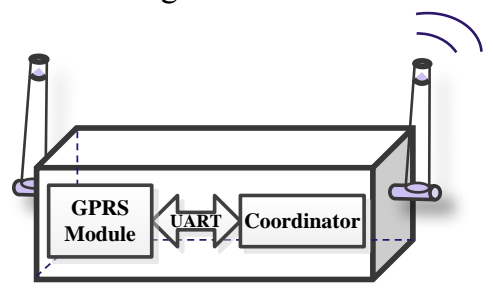

Fig.4 Diagram of intelligent gateway 


\subsubsection{Hardware circuit in the intelligent gateway}

On one hand, as the coordinator of the WSN, the gateway connected with the sensor nodes, therefore, a wireless module JN5139 was required as the MCU in the gateway. On the other hand, in order to connect with the remote server, a GPRS module was embedded in the gateway. The GPRS module was an embedded DTU ZWG-28DP GPRS (Guangzhou Zhiyuan Electronics Co.), which had the properties of compact size and flexible application. In order to realize the wireless communication in different devices, the DTU can be embedded into the users' devices easily. The DTU had a configuration interface and serial ports which can be easily used for configuration and system debugging.

\subsubsection{Software in the intelligent gateway}

The coordinator in the gateway was designed to set up the entire WSN. And the flow chart of the software in coordinator was illustrated in Fig.5. Once initializing, the coordinator searched for sensor nodes to join in and received the data sent from them. The received data were transmitted to the remote server via a GPRS module. The diagram of GPRS communication module was shown in Fig.6. A static IP was set in the remote server, and then set parameters in DTU so as to connect the remote server. The DTU connected to the coordinator via a serial port. Therefore, the coordinator connected to the remote server easily.

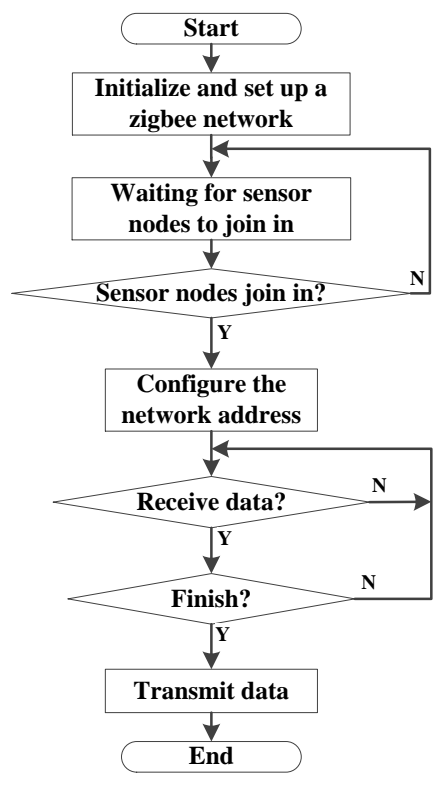

Fig.5 Flow chart of the software in coordinator

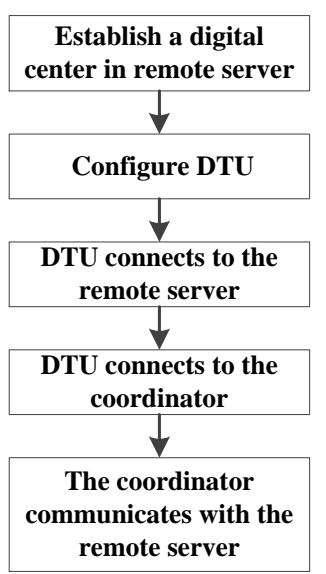

Fig.6 Diagram of GPRS communication module 


\subsection{Software in remote server}

According to the principle of the GPRS communication module shown in Fig.6, parameters of remote server and the DTU in the gateway were set. A vegetation index such as NDVI could be immediately calculated in the software once the data was transmitted to the remote server. Fig.7 showed the interface of the software, it mainly included a log-in page and a data acquisition system. And the flow chart of the software in remote server was illustrated in Fig.8.

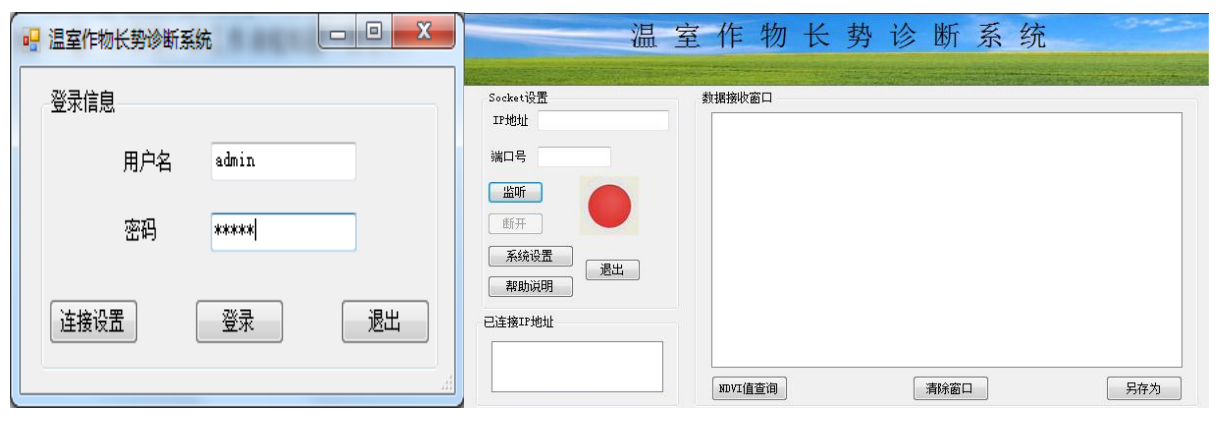

Fig.7 Interface of the software in remote server

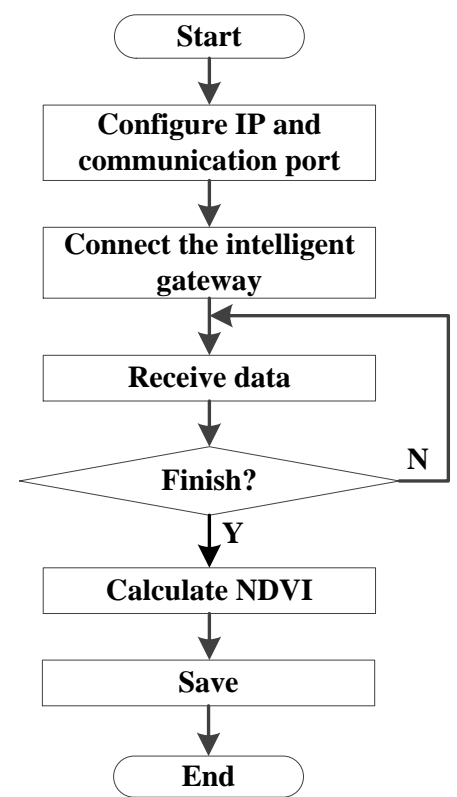

Fig.8 Flow chart of the software in remote server 


\section{Experiments and analysis}

\subsection{Test of the wireless performance}

This experiment analyzed wireless performance by testing LQI (link quality indicator) in the different communication distance. The wireless performance was tested at an experimental field located in Changping District, Beijing. There were no obstacles between sensors and gateway in the open field. Sensor nodes and gateway were placed on the shelf of $1 \mathrm{~m}$ from the ground, and the antenna was vertical upward. Transmission quality was evaluated at distances of 10, 20, 30, 40, 50, 60, 70, 80, 90, $100,110,120$ and $140 \mathrm{~m}$. Table. 1 showed the packet loss rate under different distances. The result showed that the signal of all tests could be transmitted precisely. It was confirmed that the wireless network could achieve the best communication quality and meet the requirements of agricultural application.

Table 1. Relationship between packet loss rate and communication distance

\begin{tabular}{ccc}
\hline Distance $(\mathrm{m})$ & $\begin{array}{c}\text { The actual receipt number } / \text { The } \\
\text { number of transmission }\end{array}$ & Packet loss rate \\
\hline 10 & $100 / 100$ & 0 \\
20 & $99 / 100$ & 0.01 \\
30 & $100 / 100$ & 0 \\
40 & $100 / 100$ & 0 \\
50 & $100 / 100$ & 0 \\
60 & $100 / 100$ & 0 \\
70 & $100 / 100$ & 0 \\
80 & $100 / 100$ & 0 \\
90 & $100 / 100$ & 0 \\
100 & $100 / 100$ & 0 \\
110 & $100 / 100$ & 0 \\
120 & $100 / 100$ & 0 \\
140 & $97 / 100$ & 0.03 \\
\hline
\end{tabular}

\subsection{Calibration of the optical performance}

Calibration was carried out in the campus of China Agricultural University. The sensor nodes were used to measure the incident sunlight and the reflected light of a standard white panel while an illuminometer was used to measure the sunlight. This 
panel was made of polytetrafluoroethylene (Anhui Institute of Optics and Fine Mechanics, China) and assumed to have $100 \%$ of relative reflectivity. Both illuminometer and standard white panel were set in a horizontal plane. The tests were carried out every 10 minutes from 9:00 am to 2:00 pm. Data output from illuminometer and from the signal of each optical channel were compared respectively. The result was shown in Table.2. The minimum $\mathrm{R}^{2}$ between illuminometer and each optical channel of the sensors was 0.919. It was showed that the developed sensor was sensitive to measure the sunlight (Upward) and reflected light from objects (Downward).

Table.2 Correlation coefficients between each channel of the detector and the illuminometer

\begin{tabular}{ccccc}
\hline & $550 \mathrm{~nm}$ & $650 \mathrm{~nm}$ & $766 \mathrm{~nm}$ & $850 \mathrm{~nm}$ \\
\hline Incident channels & 0.936 & 0.952 & 0.956 & 0.919 \\
Reflecting channels & 0.975 & 0.940 & 0.946 & 0.962 \\
\hline
\end{tabular}

\subsection{Experiment in greenhouse}

In order to test the effectiveness and stability of the system, the strawberry planted in greenhouse was detected. Several experiments were conducted in the greenhouse of ZhuoZhou Experiment Station in Hebei. Two sensor nodes were placed in the greenhouse to acquire the canopy spectral data of strawberries, an intelligent gateway was placed in the greenhouse as well to connect the sensor nodes and the remote server, the intelligent gateway was placed in the laboratory in China Agricultural University to receive the spectral data transmitting from the greenhouse.

According to the different combination of visible light and near infrared, software in the remote server calculated the Normalized Difference Vegetation Index (NDVI) as a detection parameter of strawberry nutrition content. The calculation formula of NDVI [12] was as follows:

$$
N D V I=\frac{R_{\mathrm{nir}}-R_{\mathrm{r}}}{R_{\mathrm{nir}}+R_{\mathrm{r}}}
$$

In the formula, $R$ nir means near-infrared spectral reflectance, and $R$ r means visible light reflectance.

The four optical channel of each sensor node can measure the waveband of $550 \mathrm{~nm}$, $650 \mathrm{~nm}, 766 \mathrm{~nm}$ and $850 \mathrm{~nm}$. NDVI can be calculated according to the different combinations of different wavebands. Results showed that from November 2013 to May 2014, during the growth stages of strawberry, the system was able to collect canopy spectral data of strawberries stably, and based on the combination of different wavebands, NDVI were calculated easily, which was used to judge the growth of strawberries. Therefore, the system provides a kind of technical support and theoretical basis for crop estimation in greenhouse 


\section{Conclusions}

Based on spectral analysis technology, electronic technology, and WSN technology, a system was developed for the crop growth estimation in greenhouse. The system was mainly made up of several intelligent sensor nodes, an intelligent gateway and software in remote server. After performance test, calibration and greenhouse experiments, the following conclusions were obtained.

(1) The sensor nodes were compact and small sized. Transmission quality of the sensor nodes was evaluated at different distances and the signal could be transmitted precisely with low packet loss rate in the tests. It was confirmed that the wireless network could achieve the best communication quality and meet the requirements of agricultural application.

(2) Calibration experiments showed that the accuracy of the optical components was high enough for application. The measured values between the monitor and illuminometer had a good correlation, and the minimum R2 between illuminometer and each optical channel of the sensors was 0.919 .

(3) The result of the greenhouse experiment showed that the system was able to collect canopy spectral data of strawberries stably during the entire growth stages of strawberry, and based on the combination of different wavebands, NDVI were calculated easily to reflect the growth of strawberry. Therefore, the system provides a kind of technical support and theoretical basis for crop estimation in greenhouse.

\section{Acknowledgment}

This research was supported by NSFC Program (31271619), the Doctoral Program of Higher Education of China (Grant No.20110008130006) and Chinese National Science and Technology Support Program (2012BAH29B04).

\section{References}

1. Mistele B, Schmidhalter U. Estimating the nitrogen nutrition index using spectral canopy reflectance measurements[J]. European Journal of Agronomy, 2008, 29(4): 184-190.

2. Guo Jianhua, Zhao Chunjiang, and Wang Xiu. Research advancement and status on crop nitrogen nutrition diagnosis[J]. Soils and Fertilizers Sciences in China, 2008, (4): 10-14.

3. Li Minzan., Han Donghai, and Wang Xiu. Spectral Analyzing technique and Applications. Science Press, Beijing, China. 2006, 177-194.

4. Filella I, Serrano L, Serra J, et al. Evaluating wheat nitrogen status with canopy reflectance indices and discriminant analysis[J]. Crop Science, 1995, 35(5): 1400-1405.

5. Xue Lihong, Cao Weixing, Luo Weihong, et al. Monitoring leaf nitrogen status in rice with canopy spectral reflectance[J]. Agronomy Journal, 2004, 96(1): 135-142.

6. Stone M L, Needham D, Solie J B, et al. Optical spectral reflectance sensor and controller: U.S. Patent 6,596,996[P]. 2003-7-22.

7. Ni Jun, Yao Xia, Tian Yongchao, et al. Design and experiments of portable apparatus for plant growth monitoring and diagnosis. Transactions of the Chinese Society of Agricultural Engineering. 2013, 29(6): 150-156. 
8. Zhang Xijie, Li Minzan, Cui Di, et al. New method and instrument to diagnose crop growth status in greenhouse based on spectroscopy[J]. Spectroscopy and Spectral Analysis, 2006, 26(5): 887-890.

9. Li Xiuhua, Li Minzan, and Cui Di. Non-destructive crop canopy analyzer based on spectral principle. Transactions of the Chinese Society for Agriculture Machinery. 2009, 40(Supplement): 252-255.

10. Li Xiuhua, Zhang Feng, Li Minzan, et al. Design of a Four-waveband Crop Canopy Analyzer. Transactions of the Chinese Society for Agricultural Machinery. 2012, 42(11): 169-173.

11. Sui, R., J. Wilkerson, W. Hart, L. Wilhelm, and D. Howard. Multi-spectral sensor for detection of nitrogen status in cotton. Applied engineering in agriculture. 2005, 21(2): 167172.

12. Yang Shuping. A measuring technology for normalized difference vegetation index[J]. Journal of Basic Science and Engineering, 2004, 12(3): 328-332. 